\title{
OLD PUZZLE, NEW INSIGHTS: A LITHIUM-RICH GIANT QUIETLY BURNING HELIUM IN ITS CORE*
}

\author{
V. Silva Aguirre ${ }^{1}$, G. R. Ruchti ${ }^{2}$, S. HeKker $^{3,4}$, S. CAssisi ${ }^{5}$, J. Christensen-DalsgaArd $^{1}$, A. Datta ${ }^{6}$, A. Jendreieck $^{7}$, \\ J. Jessen-Hansen ${ }^{1}$, A. Mazumdar ${ }^{8}$, B. Mosser ${ }^{9}$, D. Stello ${ }^{10}$, P. G. Beck ${ }^{11}$, and J. De Ridder ${ }^{11}$ \\ ${ }^{1}$ Stellar Astrophysics Centre, Department of Physics and Astronomy, Aarhus University, Ny Munkegade 120, DK-8000 Aarhus C, Denmark \\ ${ }^{2}$ Lund Observatory, Department of Astronomy and Theoretical Physics, Box 43, SE-221 00 Lund, Sweden \\ ${ }^{3}$ Max-Planck-Institut für Sonnensystemforschung, Justus-von-Liebig-Weg 3, D-37077 Göttingen, Germany \\ ${ }^{4}$ Astronomical Institute "Anton Pannekoek," University of Amsterdam, Science Park 904, 1098 HX Amsterdam, The Netherlands \\ ${ }^{5}$ INAF-Astronomical Observatory of Teramo, Via M. Maggini sn, I-64100 Teramo, Italy \\ ${ }^{6}$ Department of Physics and Meteorology, Indian Institute of Technology, Kharagpur 721302, India \\ ${ }^{7}$ Max-Planck-Institut für Astrophysics, Karl Schwarzschild Strasse 1, D-85748 Garching, Germany \\ ${ }^{8}$ Homi Bhabha Centre for Science Education, TIFR, V. N. Purav Marg, Mankhurd, Mumbai 400088, India \\ ${ }^{9}$ LESIA Observatoire de Paris, CNRS, Université Pierre et Marie Curie, Université Denis Diderot, F-92195 Meudon Cedex, France \\ ${ }^{10}$ Sydney Institute for Astronomy, School of Physics, University of Sydney, NSW 2006, Australia \\ ${ }^{11}$ Instituut voor Sterrenkunde, KU Leuven, Celestijnenlaan 200D, B-3001 Leuven, Belgium \\ Received 2013 December 17; accepted 2014 February 22; published 2014 March 10
}

\begin{abstract}
About $1 \%$ of giant stars have been shown to have large surface Li abundances, which is unexpected according to standard stellar evolution models. Several scenarios for lithium production have been proposed, but it is still unclear why these Li-rich giants exist. A missing piece in this puzzle is the knowledge of the exact stage of evolution of these stars. Using low- and-high-resolution spectroscopic observations, we have undertaken a survey of lithium-rich giants in the Kepler field. In this Letter, we report the finding of the first confirmed Li-rich core-helium-burning giant, as revealed by asteroseismic analysis. The evolutionary timescales constrained by its mass suggest that Li production most likely took place through non-canonical mixing at the RGB tip, possibly during the helium flash.
\end{abstract}

Key words: asteroseismology - stars: abundances - stars: individual (KIC 5000307) - stars: late-type stars: oscillations

Online-only material: color figures

\section{INTRODUCTION}

Lithium nuclei are readily destroyed via proton capture when they are exposed to temperatures exceeding $\sim 2.6 \times 10^{6} \mathrm{~K}$. As a star leaves the main-sequence phase, its surface $\mathrm{Li}$ abundance is expected to decrease due to the inward penetration of the convective envelope called the first dredge-up (FDU; e.g., Salaris et al. 2002). This process carries material from the surface to hotter interior regions where lithium is burned, depleting its amount compared to the initial value (Iben 1967). As a consequence, the expected canonical surface lithium abundance of a $1.5 M_{\odot}$ star at the end of the FDU is $A(\mathrm{Li}) \sim 1.5^{12}$ (e.g., Palmerini et al. 2011).

Nevertheless, about $1 \%$ of giant stars show an unusual enhancement in their surface Li abundance (cf. Brown et al. 1989), an occurrence that challenges standard stellar evolution models. A variety of scenarios have been proposed to explain this phenomenon (e.g., Sackmann \& Boothroyd 1999; Boothroyd \& Sackmann 1999; Romano et al. 1999; Charbonnel \& Balachandran 2000), commonly featuring Li enrichment during the asymptotic-giant branch (AGB) phase or at the luminosity function bump in the red giant branch (RGB). Evolutionary classifications of many known Li-rich giants, primarily based on their location in the Hertzsprung-Russell diagram (HRD), have been found consistent with this assumption.

However, observations have revealed the existence of Li-rich giants at different luminosities along the RGB (e.g., Monaco

\footnotetext{
* Based on observations made with the Nordic Optical Telescope, operated by the Nordic Optical Telescope Scientific Association at the Observatorio del Roque de los Muchachos, La Palma, Spain, of the Instituto de Astrofísica de Canarias.

${ }_{12} A(\mathrm{Li})=\log (N(\mathrm{Li}) / N(\mathrm{H}))+12$.
}

et al. 2011), whereas any lithium enhancement taking place at the bump phase is expected to be depleted by the time the star reaches the RGB tip. Moreover, Kumar et al. (2011) found Li-rich giants having atmospheric properties in agreement with them being in the clump phase (see also Martell \& Shetrone 2013). Finding Li-rich giants in the core-helium burning phase is evidence of a different enrichment scenario that could be triggered by non-canonical mixing at the RGB tip (e.g., during core helium ignition) or at the clump phase itself.

If we aim at unveiling the processes that enhance lithium in red giants, detailed elemental abundances from high-resolution spectroscopy and a clear classification of the evolutionary phase of these stars are absolutely critical. This is now possible thanks to asteroseismic observations from space-borne missions, which allow discrimination between red giants in the hydrogen-shell or core-helium burning phases (Bedding et al. 2011). A lithiumrich giant below the RGB-bump luminosity has recently been found by Anthony-Twarog et al. (2013) in the Kepler field. In this Letter, we report the discovery of the first confirmed Li-rich clump red giant star, KIC 5000307. We determine the combined spectroscopic and asteroseismic properties of this target, and use these data to investigate the mixing and nucleosynthesis processes that could originate its $\mathrm{Li}$ enhancement.

\section{CANDIDATE SELECTION AND OBSERVATIONS}

As Li-rich giants are extremely rare, large spectroscopic surveys are ideal for their identification. The Large Sky Area Multi-Object Fiber Spectroscopic Telescope (LAMOST; Cui et al. 2012) is well-suited for the discovery of these rare objects, since its low-resolution $(R \sim 2000)$ spectra has a wavelength coverage containing the $6708 \AA \mathrm{Li}$ line. Using synthetic spectra, 
Table 1

Fundamental Properties of KIC 5000307

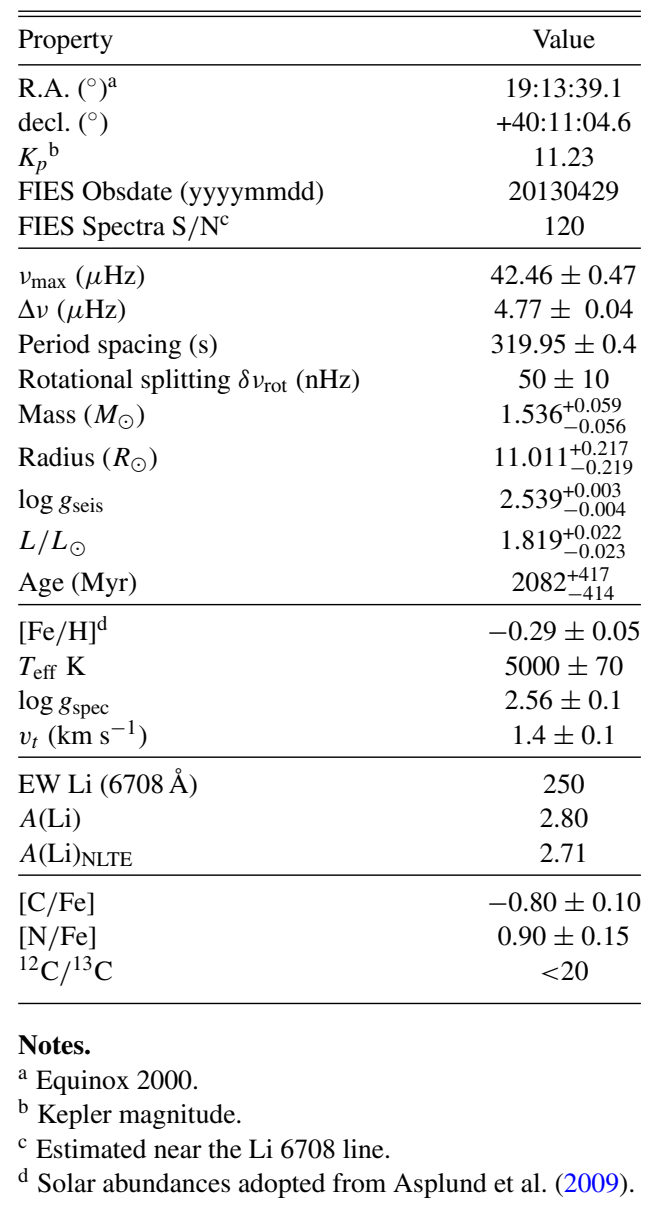

degraded to the resolution of LAMOST, we found that a $\mathrm{Li} 6708$ line with an equivalent width $W_{\lambda}>200 \mathrm{~m} \AA$ is detectable. We used the LAMOST observations of the Kepler field to search for potential Li-rich candidates. To confirm the $\mathrm{Li}$ abundance and derive more accurate stellar parameters, we obtained high-resolution spectroscopic observations using the Fibre-fed Echelle Spectrograph (FIES) on the Nordic Optical Telescope located at La Palma Observatory in the Canary Islands, Spain. We set FIES to deliver a resolving power of $R \sim 46,000$ with a spectral coverage of 3700-7300 (all spectra were reduced using the FIEStool ${ }^{13}$ reduction software).

\section{SPECTROSCOPIC ANALYSIS}

All parameters of KIC 5000307 derived directly from spectroscopic and asteroseismic observations, as well as those determined in combination with stellar models, are listed in Table 1.

\subsection{Atmospheric Parameters}

The spectroscopic stellar parameters (effective temperature, surface gravity, and metallicity) were determined following the iterative methodology described in Ruchti et al. (2013), which uses on-the-fly non-LTE (NLTE) corrections to Fe I lines. Briefly, the spectral analysis was performed with the MOOG program (Sneden 1973), using one-dimensional, planeparallel Kurucz model atmospheres (Castelli \& Kurucz 2004 and

\footnotetext{
13 http://www.not.iac.es/instruments/fies/fiestool/FIEStool.html
}

references therein), which are computed under the assumption of local thermodynamic and hydrostatic equilibrium. The effective temperature, $T_{\text {eff }}$, was derived from the wings of the Balmer lines through profile fits to $\mathrm{H} \alpha$ and $\mathrm{H} \beta$. The microturbulence was found by minimizing the slope of the relationship between the NLTE-corrected abundance of iron from $\mathrm{Fe}$ I lines and the reduced $W_{\lambda}$. The surface gravity, $\log g$, and metallicity, $[\mathrm{Fe} / \mathrm{H}]$, were then derived by minimizing the difference between the abundance of iron from the NLTE-corrected Fe I lines and that from the Fe II lines. We note that the spectroscopic $\log g$ value agrees well with the one derived using asteroseismology (see Section 4 below).

\subsection{Lithium and CN Abundances}

The ${ }^{7} \mathrm{Li}$ abundance was determined following the methodology described in Ruchti et al. (2011). We measured an equivalent width of $250 \mathrm{~m} \AA$ for the $6708 \AA \mathrm{Li}$ line. Using this value, we derived a $\mathrm{Li}$ abundance of $A(\mathrm{Li})=2.80$ in $\mathrm{LTE}$ and $A(\mathrm{Li})=2.71$ in NLTE, computed following the NLTE corrections of Lind et al. (2009a). The $[\mathrm{C} / \mathrm{Fe}]$ and $[\mathrm{N} / \mathrm{Fe}]$ ratios, as well as the ${ }^{12} \mathrm{C} /{ }^{13} \mathrm{C}$ isotopic ratio, were derived from spectral synthesis of $\mathrm{CH}$ and $\mathrm{CN}$ lines (B. Plez 2011, private communication) using MOOG under molecular equilibrium. Details of the oscillator strengths and dissociation energies can be found in Ruchti et al. (2011).

The syntheses are shown in Figure 1. The resultant abundances indicate that the star is deficient in carbon, $[\mathrm{C} / \mathrm{Fe}]=$ $-0.8 \pm 0.1$, while enhanced in nitrogen, $[\mathrm{N} / \mathrm{Fe}]=+0.9 \pm 0.15$. Features sensitive to the ${ }^{12} \mathrm{C} /{ }^{13} \mathrm{C}$ ratio are all very weak. We can thus only place a limit of ${ }^{12} \mathrm{C} /{ }^{13} \mathrm{C}<20$ using the $\mathrm{CN}$ feature at $4208.3 \mathrm{~A}$. Unfortunately, all oxygen lines are affected by telluric emission which prevented us from determining the oxygen abundance.

\section{ASTEROSEISMIC ANALYSIS}

Stars with outer convective envelopes show stochastically excited oscillations that travel across their interiors, called solarlike oscillations. Observing the frequency of these pulsations yields immediate information about the size of the cavity where these waves propagate, providing stringent constraints on its physical properties. Low- and intermediate-mass red giants exhibit this type of pulsations, clearly visible in the data obtained by the CoRoT and Kepler missions (De Ridder et al. 2009; Huber et al. 2011).

For the asteroseismic analysis we have used nearly three years of Kepler data (Q0-Q12). The Kepler light curve has been extracted using the pixel data following the methods described in S. Mathur et al. (2014, in preparation) and corrected following García et al. (2011). The power spectrum of KIC 5000307 is shown in Figure 2, where the angular degree $\ell$ describing the geometrical component of each oscillation mode is labeled. Modes of the same degree and consecutive order $n$ are approximately equally spaced in frequency, as can be seen for the $\ell=0$ case in Figure 2. This quantity is called the large frequency separation $\Delta v=v_{\ell, n}-v_{\ell, n-1}$. The overall power spectrum shows a Gaussian-shaped envelope (e.g., Chaplin \& Miglio 2013), where the frequency of maximum oscillation power is known as $v_{\max }$. These two global oscillation parameters, $\Delta v$ and $v_{\max }$, have been determined using the method described by Hekker et al. (2010).

Solar-like oscillations originate from two types of standing waves: those whose restoring force is the pressure gradient (called $p$-modes) and those where buoyancy acts as the restoring 

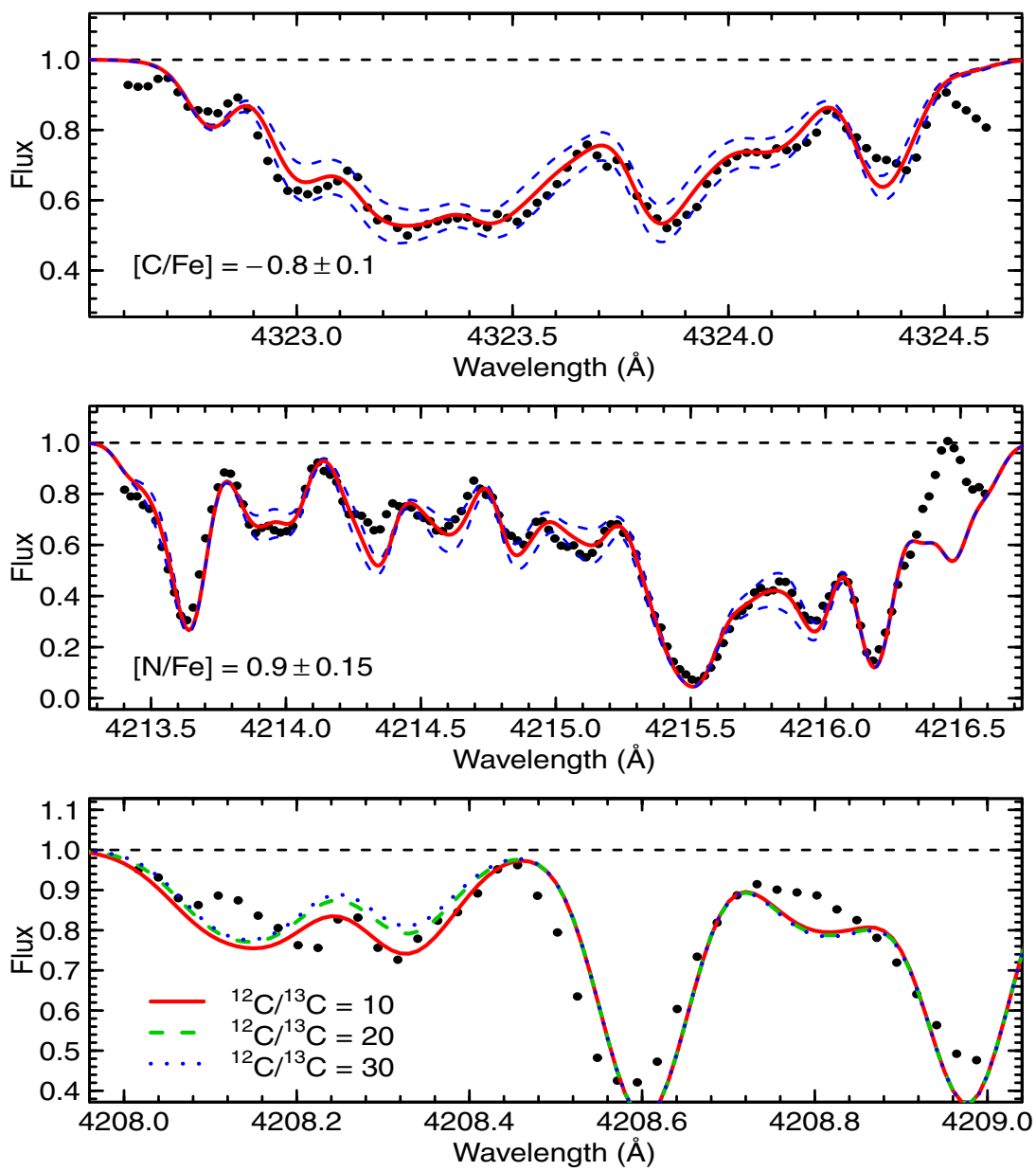

Figure 1. Resultant spectroscopic syntheses. Top: fit to the $\mathrm{CH} G$ band to estimate the carbon abundance. Black points represent the observed data. The solid, red curve shows the best fit, while the blue-dashed curves indicate the error on this fit. Middle: synthesis of the $\mathrm{CN}$ band around $4215 \AA$ to estimate [N/Fe]. The curves and points are the same as in the top plot. Bottom: synthesis of the $4208.3 \AA \mathrm{CN}$ feature. Three values of the ${ }^{12} \mathrm{C} /{ }^{13} \mathrm{C}$ ratio equal to 10,20 , and 30 are shown as a red-solid, green-dashed, and blue-dotted curve, respectively.

(A color version of this figure is available in the online journal.)

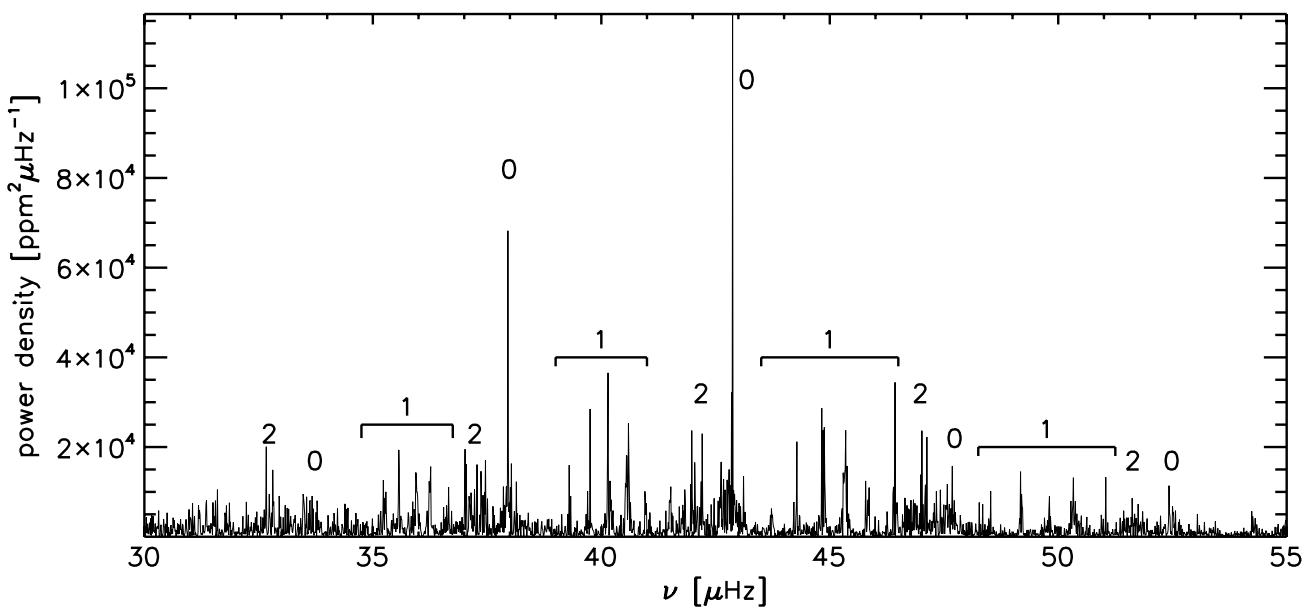

Figure 2. Power spectrum of KIC 5000307, showing the oscillation frequencies. Numbers identify peaks of equal angular degree $\ell$, with the $\ell=1$ mixed modes marked by horizontal lines.

quantity (called $g$-modes). In main-sequence stars, only pure $p$-modes reach observable amplitudes at the stellar surface, showing a very regular pattern for every angular degree (e.g., Bedding 2011). When a star leaves the main sequence, hydrogen burning ceases in the center and is restricted to a thin shell outside the inert helium core. The star's envelope expands and its core contracts, inducing a large increase in the buoyancy frequency in the center. This produces coupling between the cavities where $p$-modes and $g$-modes reside, resulting in mixed non-radial $(\ell \geqslant 1)$ modes whose pulsation frequencies behave like $p$-modes in the outer envelope and $g$-modes in the deep interior (see, e.g., Chaplin \& Miglio 2013, for a review). 


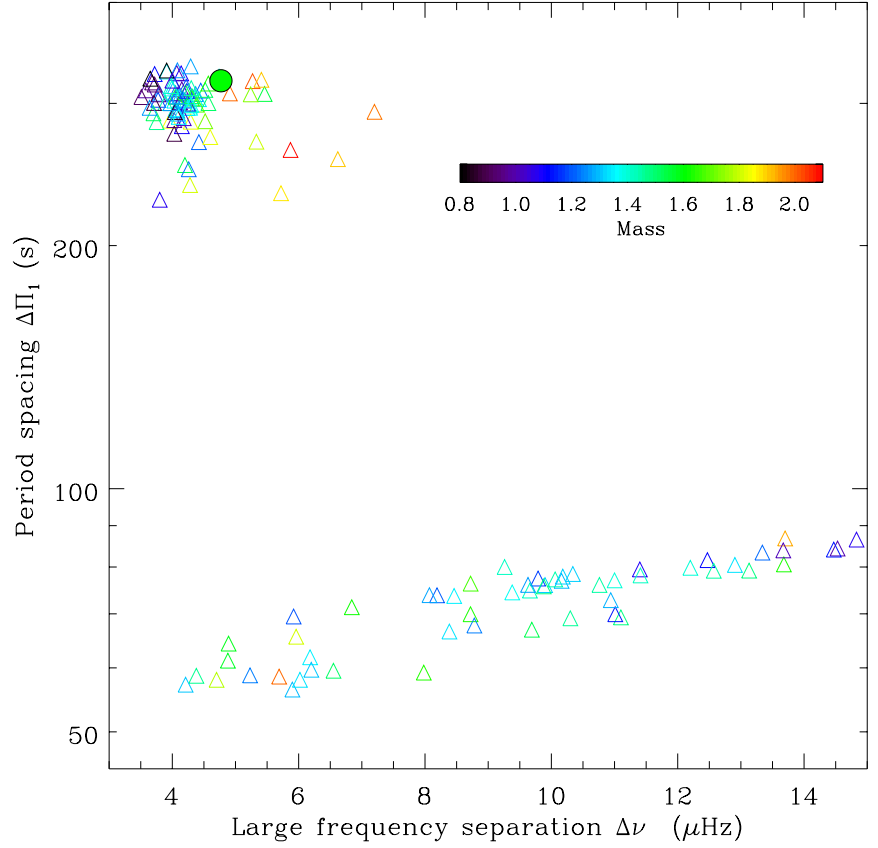

Figure 3. Sample of Kepler stars color coded by mass as determined by Mosser et al. (2012a). Stars in the RGB phase of evolution $\left(\Delta \Pi_{1} \leqslant 100 \mathrm{~s}\right)$ and the clump phase $\left(\Delta \Pi_{1} \geqslant 150 \mathrm{~s}\right)$ are clearly separated in this diagram. Location of KIC 5000307 is marked with a filled circle.

(A color version of this figure is available in the online journal.)

Red giants show a very dense spectrum of modes of mixed character, as seen in Figure 2 for the dipole $(\ell=1)$ modes. The underlying $g$-modes are approximately equally spaced in period, and measuring this separation provides strong constraints on the evolutionary stage of the star (see Figure 3 in Bedding et al. 2011). The period spacing of dipole modes $\left(\Delta \Pi_{1}\right)$ has been determined by two independently developed methods, those of Mosser et al. (2012a) and A. Datta et al. (in preparation). Briefly, the latter method uses the empirical Lorentzian variation of the observed period spacing around an underlying $p$-mode to determine the $\Delta \Pi_{1}$, or the vertical stacking in a periodéchelle diagram. Mosser et al. (2012a) on the other hand uses an asymptotic expansion of mixed modes. Regardless of the method employed, the resulting period spacing values are consistent within the uncertainties quoted in Table 1.

In Figure 3 , we show the $\Delta v$ versus $\Delta \Pi_{1}$ diagram of the Kepler sample analyzed by Mosser et al. (2012a). This figure can be used to effectively discriminate between stars in the ascending red giant branch phase and those burning helium in the core (Bedding et al. 2011; Mosser et al. 2011). Our target sits in the region where clump stars are (period spacing values above $\sim 150 \mathrm{~s}$ ), confirming that this Li-rich giant has ignited helium in its core. Combining this with its value of $\Delta v$, a proxy for the stellar mean density and thus very sensitive to the stellar radius, ensures that the star has not yet evolved toward the AGB phase (see Stello et al. 2013).

To determine the asteroseismic mass, radius, and age of KIC 5000307, we apply a technique known as the grid-based method (e.g., Stello et al. 2009; Silva Aguirre et al. 2012). Details on the tracks and equations used to determine the theoretical asteroseismic quantities can be found in Section 3 of Silva Aguirre et al. (2013). We use BaSTI isochrones (Pietrinferni et al. 2004) including the effects of core overshooting during the main-sequence and semiconvection during the clump phase, as suggested by observations of dipole modes period spacing in Kepler red giants (Montalbán et al. 2013).

The input parameters fed to the grid-based analysis are the spectroscopic temperature and metallicity, and the asteroseismic global quantities $\Delta v$ and $v_{\max }$. The method is applied using the Bayesian scheme described in Serenelli et al. (2013). Knowledge of the evolutionary stage of the target is implemented as a prior on the Bayesian probabilities that allows a precise determination of the stellar age. The asteroseismic mass of $\sim 1.5 M_{\odot}$ is consistent with a star that violently ignited helium in a flash. Our results are based in a set of isochrones not considering mass-loss in the RGB, and to include systematic effects particularly affecting the age estimate we have added in quadrature to the uncertainties the difference in the central values obtained with a set of isochrones using a Reimers (1975) mass loss rate of $\eta=0.4$.

Mosser et al. (2012b) have determined mean core rotation periods in red giants observed by the Kepler satellite. Using modes of mixed character, the authors found that stars ascending the red giant branch slightly increase their core rotation periods, while clearly spinning down in the red clump phase. The rotational splitting $\delta v_{\text {rot }}=50 \mathrm{nHz}$ measured in KIC 5000307, corresponding to a period of $\sim 100$ days and common to many other clump stars, is evidence of no particularly fast core rotation (see Figures 6 and 7 in Mosser et al. 2012b). Similarly, the predicted mean envelope rotation from the modulation of the rotational splitting is much slower than the mean core rotation (see Goupil et al. 2013).

\section{DISCUSSION}

Combining asteroseismic analysis with classical spectroscopic observations, we have confirmed the first Li-rich giant quiescently burning helium in its core. The obtained mass, radius, age, and period spacing give a consistent picture of a star that has gone through the helium flash. In Figure 4, we show its position in the HRD, together with the sample recently identified by Kumar et al. (2011). It can be seen that the lack of asteroseismic information could have resulted in KIC 5000307 being mistakenly identified as a $\sim 2 M_{\odot}$ star in the RGB phase. Knowledge of the evolutionary stage of the target allows us to test mixing hypotheses for this star.

In order to enhance the surface Li abundance, the ${ }^{7} \mathrm{Be}$ isotope (produced in the inner H-burning regions) must be quickly transported by deep circulation to the cooler upper stellar layers before decaying into lithium (Cameron \& Fowler 1971). Several physical processes have been envisaged as the non-canonical mixing mechanism capable of taking ${ }^{7} \mathrm{Be}$ to the stellar surface, such as rapid rotation (Drake et al. 2002), the interaction with a companion star (Denissenkov \& Herwig 2004), or magnetothermohaline mixing (Denissenkov et al. 2009).

In RGB stars, it is believed that the presence of a steep mean molecular weight gradient left behind by the bottom of the convective envelope at the end of the FDU prevents any extra mixing between the outer convective envelope and the hot layers where $\mathrm{H}$ burning is occurring. However, observations of the carbon isotopic ratio ${ }^{12} \mathrm{C} /{ }^{13} \mathrm{C}$ reveal that this quantity decreases below the canonical FDU value of $\sim 25$ once the star passes the RGB bump and evolves toward the RGB tip (see Lind et al. 2009b; Palmerini et al. 2011). This is a signature of some non-canonical mixing (such as thermohaline mixing, see Charbonnel \& Lagarde 2010) associated with the H-burning shell advancing in mass outward and crossing the position of maximum convective penetration. 


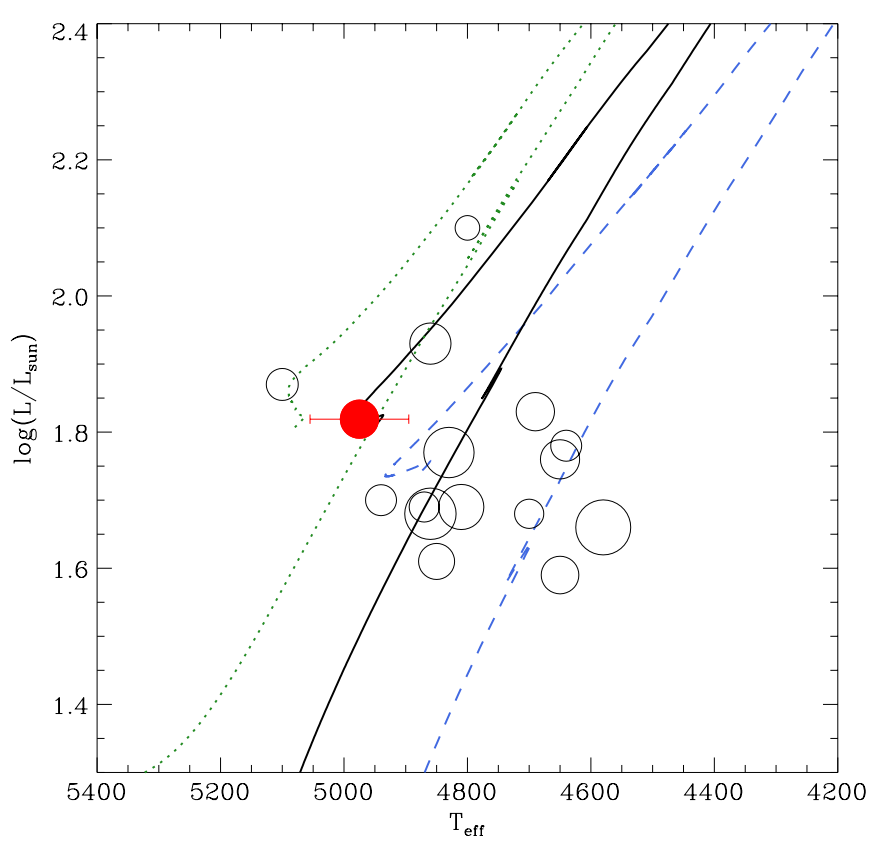

Figure 4. Position in the HRD of Li-rich giants reported in Kumar et al. (2011; open circles), while the filled symbol depicts our target KIC 5000307. Also shown are BaSTI evolutionary tracks at $[\mathrm{Fe} / \mathrm{H}]=-0.35$ for $1 M_{\odot}$ (dashed line), $1.5 M_{\odot}$ (solid line), and $2 M_{\odot}$ (dotted line). The evolution during the helium flash has been removed to avoid clutter. Symbol sizes are scaled according to $\mathrm{Li}$ abundances.

(A color version of this figure is available in the online journal.)

The low $[\mathrm{C} / \mathrm{Fe}]$ and enhanced $[\mathrm{N} / \mathrm{Fe}]$ of our target, combined with a likely non-canonical carbon isotopic ratio, describe a consistent picture of additional mixing after the FDU (possibly at the RGB bump). However, the aforementioned processes also predict a decrease in the $\mathrm{Li}$ abundance as the star evolves toward the RGB tip. Thus, even if lithium were produced at the RGB bump by these mechanisms, it should be depleted by the time the star ignites helium in its core.

Denissenkov (2012) proposed a mixing scenario in the RGB phase due to fast internal rotation and enhanced mixing across the radiative zone that could explain the lithium abundances and carbon isotopic ratios of the sample stars presented by Kumar et al. (2011). Under this prescription, after evolving past the RGB bump the star should zigzag in the HRD toward the position of the clump stars showing ${ }^{7} \mathrm{Li}$ enhancement and ${ }^{12} \mathrm{C} /$ ${ }^{13} \mathrm{C}$ depletion in its surface. After this incursion to the clump region, the star resumes its ascent toward the RGB tip before igniting helium. However, our target's $\Delta \Pi_{1}$ value is evidence of a large convective zone in the stellar center incompatible with the expected core of a RGB star. Moreover, the measured rotational splitting shows no signature of fast core or envelope rotation, ruling out the possibility of KIC 5000307 being an RGB star zigzagging toward the clump region as suggested by Denissenkov (2012).

There is no signature of binarity in the Kepler light curve nor in the spectra's radial velocity, but contamination from a companion star or planet engulfment are possible scenarios for the lithium enrichment of our target. A binary companion going through AGB thermal pulses could produce Li via the Cameron-Fowler mechanism and pollute the surface of our star. However, AGB lithium production is envisaged for masses above $\sim 3 M_{\odot}$, thus contamination would have taken place no later than $\sim 300 \mathrm{Myr}$ after the birth of the binary system and would certainly not have survived beyond the FDU in KIC 5000307.

If pollution, on the other hand, took place after the FDU it must have occurred within a narrow time span, namely, after the RGB bump and very close to the RGB tip. Given the target's mass as constrained from asteroseismology, this time frame corresponds to a few tenths of Myr. It is highly unlikely that pollution from an RGB companion, enhanced in surface lithium abundance and transferring mass to our target, would have occurred in such a short period of time.

A hypothesis first proposed by de La Reza et al. (1996) is that Li-rich giants can be identified using their far-infrared color properties. In this scenario, whatever mechanism is responsible for enhancing the surface $\mathrm{Li}$ abundance also produces the formation of a circumstellar shell of ejected material, thus affecting the photometric properties of these stars. The only far-IR photometry available for KIC 5000307 comes from the WISE catalog (Cutri et al. 2012), which unfortunately has a large uncertainty in the $22 \mu \mathrm{m} \mathrm{W4}$ color and is not conclusive about the presence of IR excess. Further observations in this region of the spectrum would be highly valuable to validate this scenario.

Another possibility is that lithium appeared in the stellar surface as a consequence of non-canonical mixing during helium ignition. Previous studies of the He-flash in very metal-poor lowmass stars suggested that the convective zone produced by the huge energy release of He-burning could penetrate the overlying hydrogen-rich layers (see Schlattl et al. 2001 and references therein). The resulting inward migration of protons ( $\mathrm{H}$-injection) into high-temperature regions leads to a $\mathrm{H}$-shell flash. As a consequence, when the convective envelope is deepening and merging with this $\mathrm{H}$-flash driven convective zone, the surface is enriched with a large amount of matter that has been processed in hydrogen fusion.

The occurrence of $\mathrm{H}$-injection has been found in hydrodynamic simulations of the core He-flash by Mocák et al. (2011) at solar metallicity. Mimicking the hydrodynamical simulations with a hydrostatic evolutionary code, the authors were able to reproduce $\mathrm{H}$-injection in a $1 M_{\odot}$ model with the outcome of a strong pollution of the stellar envelope. Interestingly enough, the Mocák et al. (2011) results show that the surface ${ }^{12} \mathrm{C} /{ }^{13} \mathrm{C}$ drops below $\sim 10$ while lithium is enriched to a level of $A(\mathrm{Li}) \sim 3.7$. However, they predict carbon enrichment in the surface by a factor of two or three, which we do not observe in KIC 5000307.

If non-canonical mixing during the He flash is responsible for the lithium enhancement, Li-rich core-helium burning stars should be mostly concentrated at the early clump stage since any enhancement occurred at the flash is expected to be depleted as the star evolves toward the AGB phase due to the deepening of the outer convection zone. Under that assumption, if KIC 5000307 has only recently gone through the helium flash, remnant processes of that episode could still be adjusting its interior structure and might be visible in the oscillation spectrum. In a future study, we will aim at detailed modeling of this target using individual frequencies of oscillations, as well as extending the sample of Li-rich giants in the Kepler field to get a more detailed view of Li-enrichment from the RGB to the AGB.

The authors thank the referee for the useful comments that improved the quality of the Letter, and M. Bergemann for providing profile fits of the Balmer lines. Funding for the Stellar Astrophysics Centre is provided by The Danish National Research Foundation (grant agreement No. DNRF106). 
The research is supported by the ASTERISK project (ASTERoseismic Investigations with SONG and Kepler) funded by the European Research Council (grant agreement No. 267864). Work by G.R.R. is fully supported by grant No. 20115042 from the Swedish Research Council. This work partially used data analyzed under the NASA grant NNX12AE17G and under the European Communitys Seventh Framework Program grant (FP7/2007-2013)/ERC grant agreement No. PROSPERITY and ERC grant agreement No. 338251 StellarAges.

\section{REFERENCES}

Anthony-Twarog, B. J., Deliyannis, C. P., Rich, E., \& Twarog, B. A. 2013, ApJL, 767, L19

Asplund, M., Grevesse, N., Sauval, A. J., \& Scott, P. 2009, ARA\&A, 47, 481

Bedding, T. R. 2011, in Asteroseismology, Canary Islands Winter School of Astrophysics, Vol XXII, ed. P. L. Pallé (Cambridge: Cambridge Univ. Press) (arXiv:1107.1723)

Bedding, T. R., Mosser, B., Huber, D., et al. 2011, Natur, 471, 608

Boothroyd, A. I., \& Sackmann, I.-J. 1999, ApJ, 510, 232

Brown, J. A., Sneden, C., Lambert, D. L., \& Dutchover, E., Jr. 1989, ApJS, 71, 293

Cameron, A. G. W., \& Fowler, W. A. 1971, ApJ, 164, 111

Castelli, F., \& Kurucz, R. L. 2004, in Proc. of IAU Symposium, Vol. 210, Modelling of Stellar Atmospheres, ed. N. Piskunov et al. (San Francisco, CA: ASP), poster A20

Chaplin, W. J., \& Miglio, A. 2013, ARA\&A, 51, 353

Charbonnel, C., \& Balachandran, S. C. 2000, A\&A, 359, 563

Charbonnel, C., \& Lagarde, N. 2010, A\&A, 522, A10

Cui, X.-Q., Zhao, Y.-H., Chu, Y.-Q., et al. 2012, RAA, 12, 1197

Cutri, R. M., Wright, E. L., Conrow, T., et al. 2012, Explanatory Supplement to the WISE All-Sky Data Release Products, Technical Report, 1

de La Reza, R., Drake, N. A., da Silva, L., et al. 1996, ApJL, 456, L115

Denissenkov, P. A. 2012, ApJL, 753, L3

Denissenkov, P. A., \& Herwig, F. 2004, ApJ, 612, 1081
Denissenkov, P. A., Pinsonneault, M., \& MacGregor, K. B. 2009, ApJ, 696, 1823

De Ridder, J., Barban, C., Baudin, F., et al. 2009, Natur, 459, 398

Drake, N. A., de la Reza, R., da Silva, L., \& Lambert, D. L. 2002, AJ, 123, 2703

García, R. A., Hekker, S., Stello, D., et al. 2011, MNRAS, 514, L6

Goupil, M. J., Mosser, B., Marques, J. P., et al. 2013, A\&A, 549, A75

Hekker, S., Broomhall, A.-M., Chaplin, W. J., et al. 2010, MNRAS, 402, 2049

Huber, D., Bedding, T. R., Stello, D., et al. 2011, ApJ, 743, 143

Iben, I. 1967, ApJ, 147, 624

Kumar, Y. B., Reddy, B. E., \& Lambert, D. L. 2011, ApJL, 730, L12

Lind, K., Asplund, M., \& Barklem, P. S. 2009a, A\&A, 503, 541

Lind, K., Primas, F., Charbonnel, C., Grundahl, F., \& Asplund, M. 2009b, A\&A, 503,545

Martell, S. L., \& Shetrone, M. D. 2013, MNRAS, 430, 611

Mocák, M., Siess, L., \& Müller, E. 2011, A\&A, 533, A53

Monaco, L., Villanova, S., Moni Bidin, C., et al. 2011, A\&A, 529, A90

Montalbán, J., Miglio, A., Noels, A., et al. 2013, ApJ, 766, 118

Mosser, B., Barban, C., Montalbán, J., et al. 2011, A\&A, 532, 86

Mosser, B., Goupil, M. J., Belkacem, K., et al. 2012a, A\&A, 540, 143

Mosser, B., Goupil, M. J., Belkacem, K., et al. 2012b, A\&A, 548, A10

Palmerini, S., Cristallo, S., Busso, M., et al. 2011, ApJ, 741, 26

Pietrinferni, S., Cassisi, S., Salaris, M., \& Castelli, F. 2004, ApJ, 612, 168

Reimers, D. 1975, MSRSL, 8, 369

Romano, D., Matteucci, F., Molaro, P., \& Bonifacio, P. 1999, A\&A, 352, 117

Ruchti, G. R., Bergemann, M., Serenelli, A., Casagrande, L., \& Lind, K. 2013, MNRAS, 429, 126

Ruchti, G. R., Fulbright, J. P., Wyse, R. F. G., et al. 2011, ApJ, 743, 107

Sackmann, I.-J., \& Boothroyd, A. I. 1999, ApJ, 510, 217

Salaris, M., Cassisi, S., \& Weiss, A. 2002, PASP, 114, 375

Schlattl, H., Cassisi, S., Salaris, M., \& Weiss, A. 2001, ApJ, 559, 1082

Serenelli, A. M., Bergemann, M., Ruchti, G., \& Casagrande, L. 2013, MNRAS, 429,3645

Silva Aguirre, V., Basu, S., Brandão, I., et al. 2013, ApJ, 796, 141

Silva Aguirre, V., Casagrande, L., Basu, S., et al. 2012, ApJ, 757, 99

Sneden, C. 1973, ApJ, 184, 839

Stello, D., Chaplin, W. J., Bruntt, H., et al. 2009, ApJ, 700, 1589

Stello, D., Huber, D., Bedding, T. R., et al. 2013, ApJL, 765, L41 